\section{THU0209 4-PHENYLBUTYRIC ACID AMELIORATES LUPUS HEPATITIS AND NEPHRITIS THROUGH SUPPRESSION OF NF-KB ACTIVATION IN EXPERIMENTAL LUPUS MODEL}

Yun-Jung Choi ${ }^{1}$, Ji-Hyun Jeong ${ }^{1}$, Eun-Kyeong Lee ${ }^{1}$, Chang-Hoon Lee ${ }^{2}$, MyeungSu Lee ${ }^{2}$, Wan-Hee Yoo ${ }^{1} .{ }^{1}$ Chonbuk National University Hospital, Internal Medicine, Jeon-Ju, Korea, Rep. of (South Korea); ${ }^{2}$ Wonkwang University Hospital, Internal Medicine, Ik-San, Korea, Rep. of (South Korea)

Background: Systemic lupus erythematosus (SLE) is an autoimmune disease presenting diverse manifestation involving multiple organs, such as liver and kidney. Endoplasmic reticulum (ER) stress has been revealed as the one of the contributing factors of lupus pathogenesis.

Objectives: The purpose of the present study was to investigate whether ER stress inhibition suppresses organ inflammation including liver and kidney in lupus murine model and the activation of mitogen activated protein kinases (MAPK) and NF-kB.

Methods: A murine lupus model was induced through a 4-week treatment with Resiquimod, a toll-like receptor agonist 7 . From the $8^{\text {th }}$ week, the mice were treated with phosphate buffered saline, 4-phenylbutyric acid (4PBA), and dexamethasone for 4 weeks. The increment of body weight, liver weight, inflammation mediator level, and the pathology of hepatitis and nephritis were analyzed at 12 weeks of age. The level of phosphorylated MAPK expression and activation of NF-kb were also evaluated.

Results: 4-PBA-treated group showed lower level of body weight increment with liver to body weight ratio compared with vehicle-treated group. 4-PBA group showed decreased inflammatory cell infiltration and fibrosis in the histologic finding of liver and kidney and lower level of inflammatory mediators, including TNF- $\alpha$ and IL-6, compared to vehicle-group. GRP78 and CHOP expression was decreased in the spleen of 4-PBA treated mice compared to vehicle-treated mice and 4-PBA group showed the lower expression level of phosphorylated JNK, ERK, p38 and NF-kB of the spleen.

Conclusion: Our results suggest that 4-PBA attenuates the inflammation on liver and kidney of experimental lupus model through suppression of MAPK and NF-kB activation. Thus, inhibition of ER stress could be function as anti-inflammatory therapeutics for SLE.
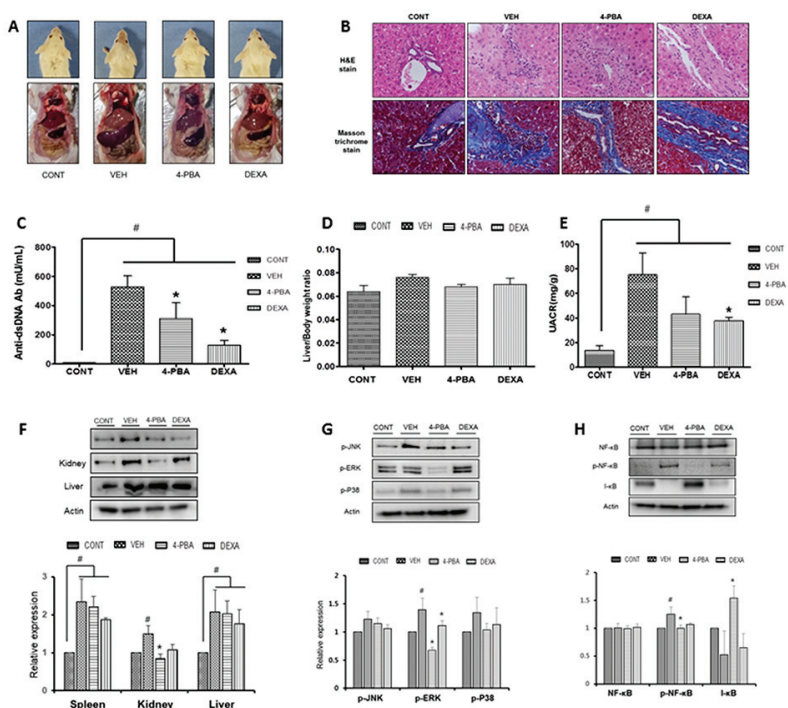

Abstract THU0209 - Figure 1

\section{REFERENCES:}

[1] Navid F, Colbert RA. Causes and consequences of endoplasmic reticulum stress in rheumatic disease. Nat Rev Rheumatol 2017;13:25-40

[2] Hosoi T, Ozawa K. Endoplasmic reticulum stress in disease: mechanisms and therapeutic opportunities. Clin Sci (Lond) 2009;118:19-29

Disclosure of Interests: None declared DOI: 10.1136/annrheumdis-2019-eular.5589

\section{THU0210 RNA-SEQ REVEALS THE MECHANISM OF THALIDOMIDE IN LUPUS CUTANEOUS LESIONS}

Cristina Solé-Marcé ${ }^{1}$, Ana Maria Alvarez-Rios ${ }^{1}$, Teresa Moline ${ }^{2}$, Berta Ferrer², Josep Ordi-Ros ${ }^{1}$, Josefina Cortés-Hernández ${ }^{1}$. 'Vall Hebron Institute Research, Barcelona, Spain; ${ }^{2}$ Hospital Vall Hebron, Barcelona, Spain

Background: Cutaneous Lupus Erythematosus (CLE) is common, largely heterogeneous and characterized by a chronic relapsing course. As many as 70 to $80 \%$ of patients with SLE will develop skin lesions at some point during the course of their disease, with a significant proportion being disfiguring and debilitating [1]. Conventional therapy consists of topical steroids and antimalarial agents but $40 \%$ of patients will be refractory to this regimen [2]. Thalidomide has been the only one that has shown an effectiveness of $90 \%$ [3], however, its mechanism of action in the disease is not known at all. In addition, its use is limited due mainly to its side effects such as teratogenicity and the development of peripheral polyneuropathy.

Objectives: Identification of the possible mechanisms of thalidomide in cutaneous lupus erythematosus.

Methods: Skin biopsies before and during treatment has been performed on a cohort of CLE patients treated $(\mathrm{N}=20)$ and not treated with thalidomide $(\mathrm{N}=5)$. Through a differential study of gene expression with RNAseq and its subsequent validation, the mechanism of thalidomide action has been identified. The cell population in the tissue and in the blood of the patients and their evolution due to the treatment has also been studied by flow cytometry. In vitro experiments using isolated lupus cutaneous lymphocyte and keratinocytes has been performed to see the specific biological effect of thalidomide (Figure 1).

Results: Flow cytometry of immune cells from blood obtained pre- and post-treatment revealed a significant activation of Thelper $(p<0.001)$, a differentation towards Th2 subpopulation and an increase of natural killer after thalidomide effect. Not significant difference were observed in macrophages and dendritic cell. In addition, after RNA-seq analysis two fundamental molecular pathways has been identified in responder thalidomide treatment patients: 1) via IRF4-NFא $\beta$ pathway modulation. 2) via AMPK mTOR pathway modulation. In vitro experiments using isolated primary cells from lupus cutaneous patients demostrated that thalidomide modulate IRF4 in lymphocytes to inhibite NFא $\beta$ pathway; however, AMPK-mTOR pathway is inhibited in keratinocytes by thalidomide effect.

Conclusion: Taken together, we show that mechanism of thalidomide in CLE is dual. It might inhibited $N F \kappa \beta$ pathway by modulation of IRF4 in lymphocyte but, in the same time, might inhibited MTOR pathway by modulation of AMPK in keratinocytes.

\section{REFERENCES:}

[1] Henry J. Lee, et al. Cutaneous lupus erythematosus: Understanding of clinical features, genetic basis, and pathobiology of disease guides therapeutic strategies. Autoimmunity 2006; 39(6): 433-444.

[2] Callen JP. Update on the management of cutaneous lupus erythematosus Brit J Dermatol 2004; 151: 731-736.

[3] Cortés-Hernández $\mathrm{J}$, et al. Thalidomide in the treatment of refractory cutaneous lupus erythematosus: prognostic factors of clinical outcome. $\mathrm{Br} J$ Dermatol. 2012; 166(3):616-23.

\section{CLE Patients ( $\mathrm{N}=\mathbf{2 0}$ )}

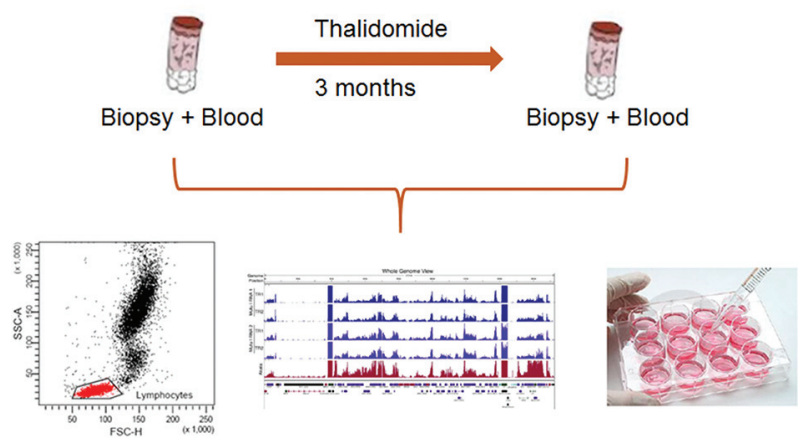

Abstract THU0210 - Figure 1. Scheme of the project to discover thalidomide mechanism in lupus cutaneous patients.

Acknowledgement: This work was financed by Instituto de Salud Carlos III (Spain Government, PI15/02145), Catalan Lupus Foundation and A.Bosch Foundation. 